\title{
PRÁTICA GERENCIAL DO ENFERMEIRO NA ESTRATÉGIA SAÚDE DA FAMÍLIA
}

\author{
NURSE MANAGEMENT PRACTICE IN THE FAMILY HEALTH STRATEGY
}

PRÁCTICA GERENCIAL DEL ENFERMERO EN LA ESTRATEGIA SALUD DE LA FAMILIA

\author{
Ludmila Mourão Xavier-Gomes ${ }^{1}$ \\ Thiago Luis de Andrade-Barbosa ${ }^{2}$ \\ Carla Silvana Oliveira Silva ${ }^{3}$ \\ Joanilva Ribeiro Lopes ${ }^{4}$ \\ Maisa Tavares de Souza Leite ${ }^{5}$
}

Resumo O presente estudo objetivou analisar a prática gerencial dos enfermeiros na Estratégia Saúde da Família. Trata-se de pesquisa descritiva e exploratória, com abordagem qualitativa pautada na fenomenologia sociológica de Alfred Schütz. Para a coleta de dados, realizou-se a entrevista semiestruturada. Os sujeitos da pesquisa foram 11 enfermeiros das equipes de saúde da família do município de Conselheiro Lafaiete, Minas Gerais, no período de agosto a novembro de 2009. Os dados coletados foram submetidos à análise de conteúdo, respeitando-se os preceitos éticos de pesquisa envolvendo seres humanos. Os resultados evidenciaram as seguintes categorias: "A percepção sobre o trabalho" e "Fatores intervenientes no trabalho". Nos discursos, destaca-se a gestão de pessoas como importante ferramenta que permeia as relações entre a equipe de saúde. Em relação aos fatores dificultadores, os profissionais levantaram questões relacionadas à insatisfação, vivenciada na falta de reconhecimento do seu trabalho assistencial e gerencial. $\mathrm{O}$ fortalecimento da ação gerencial do enfermeiro é imprescindível na construção de uma prática autônoma e ampla no contexto da atenção primária à saúde.

Palavras-chave trabalho; enfermagem; gerência; Estratégia Saúde da Família.
Abstract This study aimed to analyze the managerial practice of nurses in the Family Health Strategy. It is a descriptive and exploratory study with a qualitative approach guided by the social phenomenology of Alfred Schütz. A semi-structured interview was conducted to collect data. Study subjects were 11 nurses of family health teams in the city of Conselheiro Lafaiete, state of Minas Gerais, Brazil, in the period ranging from August to November, 2009. The data collected were subjected to content analysis, respecting the ethical principles of research involving human subjects. The results revealed these categories: "The view on work" and "Intervening factors at work." In the discourse, people management stands out as an important tool that permeates relations among the health team. Regarding hindering factors, professionals have raised issues related to dissatisfaction, experienced in the context of the lack of recognition of the work they do in providing care and management. The strengthening of nursing management action is essential in building a broad, autonomous practice in the context of primary health care.

Keywords work; nursing; management; Family Health Strategy. 


\section{Introdução}

A atenção primária à saúde representa o conjunto de ações que visam à promoção de saúde, à prevenção de agravos, ao tratamento e reabilitação nos âmbitos coletivo e individual, por meio de práticas gerenciais e sanitárias democráticas e participativas. Situa-se no primeiro nível de atenção dos sistemas de saúde, com capacidade de resolver um elenco de necessidades que extrapolam a intervenção curativa individual, denominadas necessidades básicas de saúde (Saparolli e Adami, 2010).

A valorização da temática atenção primária à saúde é observada nos planos político e acadêmico, nos âmbitos nacional e internacional, nas propostas das agências internacionais e nos processos de reformas dos sistemas de saúde nas décadas de 1980 e 1990, visando à prestação de uma assistência qualificada. Nos serviços de saúde, a qualidade é enfatizada principalmente porque o cuidado prestado ao usuário é consumido durante a sua produção, tornando-o diferente da produção de bens, na qual é possível separar o produto com defeito sem maiores consequências. Como nos demais serviços de saúde, a busca pela qualidade no trabalho de enfermagem é condição precípua para sua existência, no sentido de ser um bem produzido e consumido no ato da produção, o que o impede de ser estocado e comercializado posteriormente (Felli e Peduzzi, 2010).

No espaço em que a gerência é exercida por enfermeiro, a relação deste profissional com o usuário e com outros trabalhadores existentes no cenário da saúde imprime determinadas características ao processo de trabalho (Fernandes et al., 2010). No âmbito da Estratégia Saúde da Família (ESF), o enfermeiro exerce atividades de supervisão, treinamento e controle da equipe e atividades consideradas de cunho gerencial. No contexto sócio-histórico-político, elabora pensamentos, e a sua idealização se projeta no modo como organiza o trabalho para produção de bens e serviços. Além disso, as ações do enfermeiro devem compreender todos os níveis de atenção à saúde (promoção, proteção e recuperação), de forma articulada e integrada. Nesse contexto, o cuidado deve enfocar as principais doenças e agravos à saúde da população adscrita, que identifiquem os grupos mais suscetíveis, as faixas etárias mais atingidas, os riscos mais relevantes e os mecanismos efetivos de controle de cada caso (Passos e Ciosak, 2006).

O gerenciamento do cuidado exige do enfermeiro uma visão que envolve os valores e lógicas diferenciados impressos nas necessidades dos usuários. É preciso refletir criticamente sobre os processos de gerenciamento do cuidado para se construir uma nova realidade organizacional alinhada às práticas. Em tal contexto, espera-se que esse profissional desenvolva habilidades gerenciais em uma perspectiva participativa, na qual o objetivo é alcançado pelo esforço coletivo e não pela união de esforços indi- 
viduais. Para tanto, é importante apreender da experiência cotidiana meios de viabilizar as práticas de gestão que propiciem mudanças no modelo assistencial de saúde (Lourenção e Benito, 2010; Montezeli e Peres, 2009; Weirich et al., 2009). Diante do exposto, o presente estudo teve por objetivo analisar elementos relacionados à formação para a ação gerencial dos enfermeiros inseridos na ESF.

\section{Trajetória metodológica}

Trata-se de um estudo descritivo e exploratório 6 de natureza qualitativa fundamentada na abordagem fenomenológica, uma vez que se buscou compreender a vivência dos enfermeiros no que se refere às atividades gerenciais no âmbito da ESF. A opção por trabalhar com a fenomenologia deve-se à possibilidade de se estudar o fenômeno com base na experiência vivida pelo sujeito. No estudo, utilizou-se o referencial teórico da fenomenologia social de Alfred Schütz, a qual explica que as pessoas são movidas em função de motivações dirigidas a objetivos, em que as razões para suas ações estão marcadas pelas experiências vividas (Moreira, 2004; Capalbo, 1998).

Participaram da investigação 11 enfermeiros, lotados nas unidades da ESF situadas no município de Conselheiro Lafaiete, Minas Gerais, que aceitaram participar da pesquisa e não estavam de férias ou licença no período da coleta de dados. Os critérios de inclusão para participar da pesquisa foram: ser enfermeiro que trabalhe como gerente de enfermagem; aceitar participar da pesquisa; não estar de férias ou de licença no período da coleta de dados.

O número de participantes foi definido a partir do momento em que se percebeu que o conteúdo dos discursos revelou o fenômeno investigado e forneceu respostas às questões de pesquisa (Moreira, 2004). Foram entrevistados em sua maioria enfermeiros do sexo feminino com idade até quarenta anos, com experiência profissional variando entre um e dez anos de serviço na ESF, e todos com pós-graduação lato sensu em Saúde da Família.

A coleta de dados ocorreu no período de agosto a novembro de 2009. Inicialmente, solicitou-se autorização da Coordenação da Atenção Primária do município para o desenvolvimento das entrevistas. Para viabilizar o contato com os enfermeiros, agendaram-se encontros em que foi explicada a finalidade do estudo, seguindo-se a apresentação do termo de consentimento livre e esclarecido. Garantiu-se a autonomia dos enfermeiros em optar por participar ou não da investigação proposta.

Empregou-se como instrumento de coleta de dados a entrevista fenomenológica, na qual os sujeitos descrevem suas vivências e a forma como percebem o fenômeno. As perguntas foram orientadas por meio de um 
roteiro com questões semiestruturadas. As informações obtidas foram anotadas e gravadas e, posteriormente, transcritas pelos pesquisadores. As entrevistas ocorreram no próprio local de trabalho e tiveram uma duração aproximada de vinte a 45 minutos. O presente estudo teve como pergunta norteadora: “Como é sua prática na gerência enquanto enfermeiro inserido na ESF?"

Na perspectiva fenomenológica, os dados foram examinados em três fases: descrição, redução e compreensão. A descrição constitui o ponto-chave da fenomenologia e envolveu três elementos: a percepção, a consciência que se direciona ao mundo-vida e o sujeito pessoa, que se vê capaz de experienciar o corpo-vivido por meio da consciência.

A segunda fase da trajetória metodológica consiste na redução, cujo objetivo é selecionar as partes essenciais da descrição, isto é, somente as partes da experiência que são partes verdadeiramente da consciência, diferentemente daquelas que são suposição. Como última etapa da fenomenologia, tem-se a compreensão, que surge em conjunto com a interpretação da linguagem do sujeito. Nesse momento, existe uma tentativa de especificar o 'significado' que é essencial na descrição e na redução como forma de investigação da experiência (Moreira, 2004). Em relação à análise dos dados, utilizou-se a técnica análise de conteúdo para construção das categorias do estudo.

A pesquisa foi desenvolvida segundo os princípios éticos, com base na resolução n. 196/96 do Ministério da Saúde (Ministério da Saúde, 1996). Asseguraram-se o caráter confidencial e a privacidade por meio de atribuição de códigos de identificação aos discursos dos pesquisados.

\section{Resultados e discussão}

Estudar a prática gerencial do enfermeiro no contexto da ESF permite compreender o significado do processo de trabalho no que diz respeito à percepção dele sobre sua prática e os fatores que interferem nesse processo. Nessa perspectiva, emergiram as seguintes categorias: "A percepção sobre o trabalho" e "Fatores intervenientes no trabalho".

\section{A percepção sobre o trabalho}

Esse núcleo se constitui das reações dos enfermeiros em relação ao trabalho desempenhado na organização. Relaciona-se, portanto, aos aspectos de satisfação e de insatisfação no trabalho, bem como às expectativas ligadas à gerência.

No discurso dos enfermeiros, observa-se a experiência da gratificação do trabalho desempenhado na ESF expressa por meio de sentimentos que 
traduzem a satisfação e a realização pessoal e profissional, quando eles afirmam desenvolver um trabalho gratificante, conforme ilustra a seguinte fala:

Sinto-me muito lisonjeada, pois, desta forma, gerenciando, recebo o reconhecimento das pessoas porque me preocupo com elas (E8).

A satisfação profissional ou satisfação no trabalho podem ser conceituadas como um sentimento agradável ou estado emocionalmente positivo do trabalhador, resultante da percepção ou avaliação de sua experiência profissional, conforme metas e valores pessoais individuais perante a vida, que é modificada ou influenciada por forças internas ou externas ao trabalho (Martinez e Paraguay, 2003; Melo, Barbosa e Souza, 2011).

A satisfação também é decorrente do reconhecimento da população. Nesta pesquisa, observou-se que o reconhecimento da clientela sobre o trabalho desempenhado é dotado de significação para os entrevistados, uma vez que constitui um motivo de satisfação. Esse significado parece estar relacionado a momentos de êxito já vivenciados pelos enfermeiros, no dia a dia, pelo trabalho que desempenham na unidade de saúde na qual estão inseridos.

Ser gerente é motivo de orgulho, de valorização, apesar de algumas pessoas não reconhecerem bem o nosso trabalho (E3).

Os relatos apontam a falta de reconhecimento do trabalho não só gerencial, mas também da enfermagem:

Sinto necessidade de maior reconhecimento da profissão. Somos muito cobradas e pouco valorizadas (E2).

Os enfermeiros demonstram indícios de insatisfação ao relatarem a falta de reconhecimento do trabalho assistencial e gerencial:

É uma carga de muita responsabilidade, porque é tudo em cima da enfermeira, tendo que gerenciar tudo. Todo o administrativo e o assistencial (E1).

A responsabilidade do enfermeiro é ressaltada por alguns entrevistados. No processo de trabalho, o gerente deve se adequar a determinados mecanismos próprios da unidade de saúde que propiciam o desenvolvimento da execução da prática gerencial (Passos e Ciosak, 2006; Xavier-Gomes e Barbosa, 2011).

Sentimentos que dão indícios de insatisfação também são apreendidos nas falas dos entrevistados, nos relatos sobre o acúmulo de atividades assistenciais e administrativas. 
Em alguns casos, trabalhamos além de nossas funções (E5).

Sinto-me sobrecarregada devido aos dois trabalhos: assistencial e gerencial (E6).

Nos relatos dos enfermeiros, a sobrecarga de trabalho é referida como excesso de atividades, como o acúmulo de funções administrativas e assistenciais, por exemplo. O enfermeiro está capacitado para realizar as atividades assistenciais e gerenciais, ambas complementares. O trabalho na ESF deve ser organizado pelo gestor de maneira que as facilidades estejam presentes em todas as fases de construção, ou seja, desde a seleção da equipe multiprofissional até a consecução dos resultados, o que inclui o planejamento, a organização e a realização cotidiana do processo de trabalho (Ximenes e Sampaio, 2008).

Dentre os fatores que facilitam o trabalho gerencial do enfermeiro, destaca-se a gestão de pessoas, ou seja, as relações que permeiam a equipe de saúde, conforme evidenciado nos discursos a seguir:

Os fatores que facilitam é a boa vontade da equipe. Colaboram e agilizam as coisas que chegam na última hora (E1).

Recursos humanos adequados e localização excelente (E2).

O bom relacionamento com a equipe de um modo geral (E5).

O que me facilita é ter bom relacionamento, sabendo respeitar cada um em sua função (E7).

O reconhecimento interpessoal entre os membros da equipe (E8).

A colaboração dos membros da equipe, principalmente os agentes que ajudam na parte administrativa (E9).

A compreensão do real significado de equipe é fundamental para um atendimento em saúde, pois para que haja qualidade e eficiência na assistência prestada, é essencial a concepção coletiva do trabalho, assim como sua execução. Nesse sentido, o trabalho em equipe é um instrumento facilitador do cuidado, de modo que o desempenho e a eficiência sejam superiores aos resultados obtidos pela execução individualizada do trabalho. Para tanto, são necessários a motivação, a criatividade, o entusiasmo, a participação, o envolvimento e o comprometimento da equipe, a fim de que a qualidade do serviço seja alcançada e os clientes se encantem (Abreu et al., 2005; Hernández Ortiz e Gomez Torres, 2010). 
Outro elemento crucial da prática gerencial do enfermeiro é a autonomia. Este elemento profissional tem sido, ao longo do tempo e da evolução da enfermagem, um tema importante para a compreensão da profissão, tanto na definição de seus desafios e objetivos quanto na forma como esse profissional se relaciona com a equipe de saúde e a sociedade em geral e se apresenta para elas (Gomes e Oliveira, 2005). O enunciado a seguir revela que há compreensão equivocada dos entrevistados acerca do conceito de autonomia:

Tenho [autonomia] dentro das limitações da profissão, pois tento resolver tudo aquilo que surge dentro na minha região e setor (E7).

Dessa forma, é possível perceber que o enfermeiro, ao ser indagado sobre autonomia, relatou aspectos da hierarquia do serviço sem descrever de forma correta o conceito do termo. A autonomia profissional está diretamente relacionada à independência e à liberdade na tomada de decisão nas atividades diárias, ou ainda na efetividade do seu processo de trabalho (Linch, Guido e Fantin, 2010).

Assim, é fundamental a clareza do conceito da autonomia profissional no sentido de que o enfermeiro entenda seu papel como possuidor de conhecimentos científicos para a sua prática e os use em benefício dos seus clientes (Gomes e Oliveira, 2010). Além disso, existe a situação complexa da enfermagem em relação à sociedade, uma vez que essa área necessita demonstrar a sua importância técnica, mercadológica e social no interior da equipe de saúde, pois enfrenta a sobreposição de atividades com outros profissionais em muitas situações (Linch, Guido e Fantin, 2010).

\section{Fatores intervenientes no trabalho}

Dentre os fatores dificultadores do processo gerencial referido pelos enfermeiros, destacam-se a estrutura física inadequada da unidade e a quantidade insuficiente de recursos materiais, além da falta de logística adequada, que envolve a provisão dos recursos materiais à unidade da saúde.

O que dificulta é a infraestrutura inadequada, escassez de material, número reduzido de exames laboratoriais (E2).

A falta de material de escritório, atraso na entrega do material para o curativo e material da farmácia (E9).

As unidades de saúde da família devem apresentar uma estrutura física adequada aos diversos serviços e procedimentos, com espaços definidos e 
instalações específicas para vacinação, terapia de reidratação, verificação de medidas antropométricas, aerossolterapia, curativos, consultas de enfermagem e médicas, saúde bucal, copa, banheiros internos e externos, triagem, recepção, esterilização, guarda de material e atividades educativas (Brasil, 2002).

Se em uma unidade há concorrência de técnicas/procedimentos em um mesmo espaço físico, tal situação gera um complicador para os trabalhadores, capaz de aumentar a tensão do processo de trabalho - o que desmotiva e interfere na qualidade de vida no trabalho. O que se espera da estrutura de uma unidade da ESF não é a perfeição ou que seja padrão, mas que possa conformar, no espaço, todas as suas necessidades organizativas, assistenciais e da comunidade (Abreu et al., 2005).

Outro fator dificultador destacado pelos entrevistados é a ausência das tecnologias duras, ou seja, indisponibilidade de equipamentos tecnológicos em geral nas unidades, principalmente computadores.

A não informatização da ESF, a falta dos meios de comunicação (...) e atraso na comunicação com a Secretaria de Saúde (E11).

A falta de uma rede informatizada é considerada um grande problema na atenção primária, o qual impossibilita que os profissionais atuantes nas comunidades locais lancem os dados dos relatórios preenchidos no Sistema de Informação da Atenção Básica (Siab) e analisem as condições de saúde da população de interesse, com base em dados estatísticos e epidemiológicos (Fernandes, Machado e Anschau, 2009).

O que dificulta é a falta de programação da Secretaria e enviar tudo na última hora. Organizar e programar sem um tempo para reunir a população para uma ação educativa, sendo assim necessário tempo, e isso não acontece. É só para atender necessidades da Secretaria da Saúde (E1).

A programação de atividades da Secretaria Municipal de Saúde muitas vezes dificulta o trabalho gerencial, uma vez que não há planejamento de longo prazo. Essa falta de planejamento de longo prazo se relaciona com a padronização de atividades no local de trabalho. Observa-se, por meio dos depoimentos, que a tomada de decisões ocorre em cima da hora, assim não há como se desenvolverem as atividades propostas num curto prazo. Ressalta-se ainda que muitas atividades da ESF necessitam da mobilização da comunidade, o que poderia não acontecer em um tempo curto.

A padronização dos procedimentos por meio de protocolos e procedimentos operacionais padrão é de grande relevância para a assistência de qualidade na saúde. 
Não há uma uniformidade de atendimento entre as equipes de saúde, embora já seja projeto da Secretaria de Saúde uniformizar o atendimento. Cada ESF faz um procedimento, e quando o paciente muda acontecem conflitos (E9).

A falta de padronização e protocolos dificulta o serviço (E3).

Em sentido restrito, 'protocolo' significa algo ou alguém que se predispõe a pôr algo ou alguém como pronto a ser utilizado ou requerido de certa forma preconcebida, por meio de recursos a ele atribuídos. Ou, ainda, é a padronização de leis e procedimentos que são dispostos para a execução de uma determinada tarefa (Ferreira, 2010).

Quanto às dificuldades em relação à gestão de pessoas, um estudo (Fernandes, Machado e Anschau, 2009) sobre gerência de unidades da atenção básica observou sobrecarga de atividades muitas vezes relacionadas com recursos humanos insuficientes; pouco compromisso de alguns; pessoas que não aceitam mudanças; a visão diferente de muitos profissionais, que acabam por priorizar os interesses individuais em detrimento dos coletivos; e a dificuldade em conseguir contentar a todos, também evidenciada nos relatos a seguir:

Um fator que dificulta é quando não se tem interação da equipe, ou seja, não trabalham para alcançar um objetivo que é a melhoria do trabalho (E4).

Relacionamento interpessoal prejudicado, falta de diálogo na equipe (E5).

A falta constante do médico e o não cumprimento do horário (E9).

A interação dos membros da equipe de saúde é de fundamental importância para o desenvolvimento do trabalho na ESF, uma vez que cada membro da equipe tem suas atribuições necessárias ao serviço. Dessa forma, são necessárias algumas competências para a interação da equipe, tais como comunicação, negociação e trabalho em equipe, dentre outras.

O gerenciamento do trabalho em saúde compreende o pensar e o fazer, que englobam o ser humano, o meio ambiente e a integração dos diversos saberes. A ordem desse tempo atual é de repensar novas alternativas, não radicais e sim integrativas, que possam agregar eficiência e, consequentemente, saúde aos seus profissionais e aos clientes (Fernandes et al., 2010).

A gerência de serviços envolve competência e habilidade para trabalhar as deficiências nos processos de trabalho e exigir do sistema municipal condições de trabalho satisfatórias, que procurem motivar os profissionais atuantes nas unidades locais de saúde, a fim de prestar um atendimento qualificado e resolutivo (Fernandes, Machado e Anschau, 2009). 
A utilização do método fenomenológico para a pesquisa empírica, no entanto, apresenta limitações, uma vez que o acesso à experiência vivida pelos sujeitos de pesquisa é sempre mediado pelas referências teóricas do pesquisador, as quais orientam a própria formulação da questão da pesquisa.

\section{Considerações finais}

O estudo ora apresentado permitiu analisar a prática gerencial dos enfermeiros no contexto da ESF. Foi possível perceber a satisfação pelo reconhecimento do trabalho desenvolvido na unidade básica de saúde. Esse aspecto do trabalho constitui um dos grandes motivadores da satisfação profissional. Contudo, alguns enfermeiros demonstraram indícios de insatisfação, ao relatarem a falta de reconhecimento do seu trabalho assistencial e gerencial. Tal percepção tem se relacionado, sobretudo, ao acúmulo de atividades assistenciais e administrativas.

Em relação aos fatores facilitadores do trabalho gerencial, os enfermeiros entrevistados destacaram a gestão de pessoas como importante ferramenta que permeia as relações entre a equipe de saúde. Todavia, a estrutura física e tecnológica inadequada e a escassez de materiais são apontadas como fatores dificultadores na prática gerencial, que repercutem na qualidade e na eficiência da assistência prestada ao cliente. Além disso, a falta de programação das atividades da Secretaria Municipal de Saúde dificulta o trabalho gerencial de enfermagem no planejamento a longo prazo.

Há que se ressaltar que os enfermeiros não conseguiram descrever o conceito de autonomia aplicada à profissão, mas apenas aspectos da hierarquia do serviço. É de suma importância que o profissional enfermeiro entenda a autonomia no contexto de sua prática, no sentido de possibilitar a ele um agir independentemente de outras categorias profissionais.

A construção do conhecimento acerca da prática gerencial de enfermagem no âmbito da ESF é imprescindível no sentido de tomar decisões e inovar nas atividades desenvolvidas com a equipe e a comunidade. Some-se a isso a necessidade de identificar as deficiências no processo de trabalho a fim de prestar um atendimento qualificado e resolutivo à população.

O presente estudo pretende contribuir para o esclarecimento de fatores dificultadores relacionados pelos profissionais, como questões relacionadas à insatisfação, vivenciadas na falta de reconhecimento do seu trabalho assistencial e gerencial. Nesse contexto, ressalta-se a necessidade de novos estudos que busquem desvelar de forma mais detalhada a prática gerencial no âmbito da ESF, de modo que se favoreçam a tomada de decisões e a inovação nas atividades desenvolvidas com a equipe e a comunidade. 


\section{Colaboradores}

Todos os autores participaram da coleta, da análise dos dados e da redação do artigo.

Resumen El presente estudio tuvo por objetivo analizar la práctica gerencial de los enfermeros en la Estrategia Salud de la Familia. Se trata de una investigación descriptiva y exploratoria, con enfoque cualitativo orientado en la fenomenología sociológica de Alfred Schütz. Para la recolección de datos, se realizó la entrevista semiestructurada. Los individuos de la investigación fueron 11 enfermeros de los equipos de salud de la familia del municipio de Conselheiro Lafaiete, Minas Gerais, Brasil, en el período de agosto a noviembre de 2009. Los datos recolectados se sometieron al análisis de contenido, respetando los preceptos éticos de investigación con participación de seres humanos. Los resultados revelaron las siguientes categorías: "La percepción sobre el trabajo" y "Factores que intervienen en el trabajo". En los discursos, se destaca la gestión de personas como importante herramienta que trasunta las relaciones entre el equipo de salud. En relación a los factores obstaculizantes, los profesionales plantearon temas relacionados a la insatisfacción, vivida en la falta de reconocimiento de su trabajo asistencial y gerencial. El fortalecimiento de la acción gerencial del enfermero es imprescindible en la construcción de una práctica autónoma y amplia en el contexto de la atención primaria de la salud.

Palabras clave trabajo; enfermería; gerencia; Estrategia Salud de la Familia.

\section{Notas}

1 Universidade Federal da Integração Latino-Americana, Instituto Latino-Americano de Ciências da Vida e da Natureza, Foz do Iguaçu, Paraná, Brasil.

<ludyxavier@yahoo.com.br>

Correspondência: Avenida Francisco Caetani, 673, Major Prates, CEP 39403-202, Montes Claros, Minas Gerais, Brasil.

2 Universidade Estadual de Montes Claros, Programa de Pós-Graduação em Ciências da Saúde, Montes Claros, Minas Gerais, Brasil.

$<$ tl_andrade@yahoo.com.br>

3 Universidade Estadual de Montes Claros, Departamento de Enfermagem, Montes Claros, Minas Gerais, Brasil.

<carla_silvana@santoagostinho.edu.br>

4 Faculdades Santo Agostinho de Montes Claros, Curso de Graduação em Enfermagem, Montes Claros, Minas Gerais, Brasil.

$<$ joanilva@yahoo.com.br> 
5 Universidade Estadual de Montes Claros, Programa de Pós-Graduação em Ciências da Saúde, Montes Claros, Minas Gerais, Brasil.

$<$ mtsiv@terra.com.br>

6 O projeto de pesquisa foi aprovado pelo Comitê de Ética em Pesquisa da Faculdade Santa Rita (Fasar), Conselheiro Lafaiete, Minas Gerais, no processo n. 0038/2009. A pesquisa não teve financiamento. Os autores declaram não haver conflitos de interesse.

\section{Referências}

ABREU, Ludmila O. et al. O trabalho de equipe em enfermagem: revisão sistemática da literatura. Revista Brasileira de Enfermagem, Brasília, v. 58, n. 2, p. 203-207, 2005.

BRASIL. Ministério da Saúde. Agência Nacional de Vigilância Sanitária (Anvisa). Resolução RDC n. 50, de 21 de fevereiro de 2002. Dispõe sobre o regulamento técnico para planejamento, programação, elaboração e avaliação de projetos físicos de estabelecimentos assistenciais de saúde. Disponível em: <http://portal.anvisa.gov.br/wps/wcm/ connect/ca36b200474597459fc8df3fbc4c6735/ $\mathrm{RDC}+\mathrm{N} \% \mathrm{C} 2 \% \mathrm{BA} .+50,+\mathrm{DE}+21+\mathrm{DE}+$ FEVEREIRO+DE+2002.pdf?MOD=AJPERES $>$. Acesso em: 17 nov. 2011.

BRASIL. Ministério da Saúde. Comissão Nacional de Ética em Pesquisa. Resolução n. 196/96. Disponível em: <http://dtr2004. saude.gov.br/susdeaz/legislacao/arquivo/ Resolucao_196_de_10_10_1996.pdf > . Acesso em: 18 nov. 2011.

CAPALBO, Creusa. Metodologia das ciências sociais: a fenomenologia de Alfred Schütz. 2. ed. Londrina: Universidade Estadual de Londrina, 1998.

FELLI, Vanda E. A.; PEDUZZI, Mariana. O trabalho gerencial em enfermagem. In: KURCGANT, Paulina (org.). Gerenciamento em enfermagem. Rio de Janeiro: Guanabara Koogan, 2010. p. 1-13.
FERNANDES, Léia C. L.; MACHADO, Rebel Z.; ANSCHAU, Geovana O. Gerência de serviços de saúde: competências desenvolvidas e dificuldades encontradas na atenção básica. Ciência \& Saúde Coletiva, Rio de Janeiro, v. 14, n. esp., p. 1.541-1.552, 2009.

FERNANDES, Marcelo C. et al. Análise da atuação do enfermeiro na gerência de unidades básicas de saúde. Revista Brasileira de Enfermagem, Brasília, v. 63, n. 1, p. 11-15, 2010.

FERREIRA, Aurélio B. H. Dicionário Aurélio da lingua portuguesa. 5. ed. Curitiba: Positivo, 2010.

GOMES, Antonio M. T.; OLIVEIRA, Denize C. A representação social da autonomia profissional do enfermeiro na saúde pública. Revista Brasileira de Enfermagem, Brasília, v. 58, n. 4, p. 393-398, 2005.

GOMES, Antonio M. T.; OLIVEIRA, Denize C. Autonomia profissional em um desenho atômico: representações sociais de enfermeiros. Revista Brasileira de Enfermagem, Brasília, v. 63, n. 4, p. 608-615, 2010.

HERNÁNDEZ ORTIZ, Joana; GOMEZ TORRES, Daniela. Una aproximación al concepto de gerencia y administración aplicado a la disciplina de enfermería. Escola Ana Nery Revista de Enfermagem, Rio de Janeiro, v. 14, n. 3, p. 625-632, 2010. 
LINCH, Graciele F. C.; GUIDO, Laura A.; FANTIN, Simone S. Enfermeiros de unidades de hemodinâmica do Rio Grande do Sul: perfil e satisfação profissional. Texto \& Contexto Enfermagem, Florianópolis, v. 19, n. 3, p. $488-495,2010$.

LOURENÇÃO, Daniela C. A.; BENITO, Gladys A. V. Competências gerenciais na formação do enfermeiro. Revista Brasileira de Enfermagem, Brasília, v. 63, n. 1, p. 91-97, 2010.

MARTINEZ, Maria C.; PARAGUAY, Ana I. B. B. Satisfação e saúde no trabalho: aspectos conceituais e metodológicos. Cadernos de Psicologia Social do Trabalho, São Paulo, v. 6, n. 1, p. 59-78, 2003.

MELO, Márcia B.; BARBOSA, Maria A.; SOUZA, Paula R. Job satisfaction of nursing staff: integrative review. Revista Latino-Americana de Enfermagem, Ribeirão Preto, SP, v. 19, n. 4, p. 1.047-1.055, 2011.

MONTEZELI, Juliana H.; PERES, Aida M. Competência gerencial do enfermeiro: conhecimento publicado em periódicos brasileiros. Cogitare Enfermagem, Curitiba, v. 14, n. 3, p. 553-558, 2009.

MOREIRA, Daniel A. O método fenomenológico na pesquisa. São Paulo: Pioneira, 2004.
PASSOS, Joanir P.; CIOSAK, Suely I. A concepção dos enfermeiros no processo gerencial em unidade básica de saúde. Revista da Escola de Enfermagem da USP, São Paulo, v. 40, n. 4, p. 464-468, 2006.

SAPAROLLI, Eliana C. L.; ADAMI, Nilce Piva. Avaliação da estrutura destinada à consulta de enfermagem à criança na atenção básica. Revista da Escola de Enfermagem da USP, São Paulo, v. 44, n. 1, p. 92-98, 2010.

WEIRICH, Claci F. et al. O trabalho gerencial do enfermeiro na rede básica de saúde. Texto \& Contexto Enfermagem, Florianópolis, v. 18, n. 2, p. 249-257, 2009.

XAVIER-GOMES, Ludmila M.; BARBOSA, Thiago L. Andrade. Trabalho das enfermeiras-gerentes e a sua formação profissional. Trabalho, Educação e Saúde, Rio de Janeiro, v. 9, n. 3, p. 449-459, 2011.

XIMENES, Francisco R. G.; SAMPAIO, José J. C. Processo de ascensão ao cargo e as facilidades e dificuldades no gerenciamento do território na Estratégia Saúde da Família. Revista Brasileira de Enfermagem, Brasília, v. 61, n. 1, p. 36-45, 2008.

Recebido em 18/07/2012

Aprovado em 23/02/2015 